\title{
AUTOMATED DRIVING TEST SCORE GENERATOR USING 3D SIMULATION
}

\author{
Hrishikesh Gaikwad ${ }^{1}$, Rutuja Mandlik ${ }^{2}$, Mrigesh Dasgupta ${ }^{3}$, AkshayLoke $^{4}$ \\ ${ }^{I}$ Department of Information Technology, Vidyalankar Institute of Technology, Vidyalankar College Marg, Wadala \\ East, Mumbai, Maharashtra 400037 \\ ${ }^{2}$ Department of Information Technology, Vidyalankar Institute of Technology, Vidyalankar College Marg, Wadala \\ East, Mumbai, Maharashtra 400037 \\ ${ }^{3}$ Department of Information Technology, Vidyalankar Institute of Technology, Vidyalankar College Marg, Wadala \\ East, Mumbai, Maharashtra 400037 \\ ${ }^{4}$ Department of Information Technology, Vidyalankar Institute of Technology, Vidyalankar College Marg, Wadala
} East, Mumbai, Maharashtra 400037

\begin{abstract}
This Driving System project allows a person to get an overview of the scenarios he may face during real time driving. So the driving system is an important way of teaching as well as making the person aware of the overall scenarios and conditions he may face while driving a vehicle through a $3 D$ game. Thus using this project, we aim at reducing the number of accidents by testing the ability of the applicants regarding driving rules using a marking system. The user will have to go through a series of tests where he will be facing many real life traffic and road scenarios in a virtual environment and then will be evaluated based on the basis of his performance. The user will get a hands on experience on a driving kit which will comprise of steering wheel, accelerator and brake thus giving a feeling of driving real car.
\end{abstract}

Keywords: - 3D game, driving kit, marking system, virtual environment..

\section{INTRODUCTION}

This project presents an idea of developing a 3D game for driving practice and interfacing it with the driving kit. Thus it will provide real-time environment to the player to face different scenarios he may face during driving. We believe in a safer world, a world with quality drivers, a world with less accidental death and for this we are implementing a virtual world or we can say virtual driving simulation.

This modular and adaptable system attaches to commerciallyavailable stationary car systems and interfaces with a computer or laptop for simulation and data acquisition processes. Parameters monitored by these systems are communicated to a driver interface screen and can be amplified before entering its virtual environment. This simulation will help the people as we can simulate real life scenarios like how to get away with an accident which is not possible in traditional question answer test system. In this there will be different test cases like applicant follow the rules for example, if the speed limit is 30 $\mathrm{km} / \mathrm{hr}$. , then driver should not cross the speed limit and follow the traffic signal rules.

In recent years, the number of deaths caused by traffic accidents has been decreasing in line with advances in vehicle safety systems and emergency rescue and medical techniques. On the other hand, the number of traffic accidents still remains high. In particular, accidents involving elderly and young people have been increasing in number, not only as injured pedestrians but also as culpable drivers. It is said that one factor in this is a higher-aged society with less children.

Originally, the traffic environment was divided into two kinds of structures, such as a strong site like a vehicle driver and a vulnerable site like a pedestrian. The former needs sufficient ability to control the vehicle, and therefore they must have gained a license through an aptitude test for driving ability. On the other hand, the latter does not need the same kind of ability as drivers. However, the person in both sites in the traffic environment must understand the safety rules and manners.

\section{AIMS AND OBJECTIVES}

The objective of this project is to yield better drivers. Our project aims at educating the users about the traffics rules and regulations and also giving them a hands-on experience about driving in the Virtual world.

The driver will be facing various test cases during the course of his examination which will judge how he faces real life situations. The driver has to follow all the traffic rules and regulations of the road so that he gets used to the practical environment.

Our project will consist of a marking system where the user will be marked based on how he performs in the test. There will be an initial score to start with and as he makes 
progress through the test, certain marks will be deducted if he commits a mistake. At the end of the test, result will be displayed to show whether he has passed or failed. If we commit more than three mistakes or breaks the laws then he will be failed.

The Test will help the user to get the knowledge about driving in an real environment and also make him ready to drive the car in real world.

\section{LITERATURE SURVEY}

\subsection{UNITY Game}

The people at OTEE and David Janik-Jones in their research paper Unity racing Game tutorial have explained how to create a complete racing game. This paper has introduced more advanced concepts and techniques in Unity, and have focused on scripting advanced functions within the game. The have also gradually build a complete game following easy step-bystep instructions and progression of concepts.

\subsection{Virtual Environment Design}

Chi-Wen Yang, Tsung-HanLee, Chien- Lung Huang and Kuei-Shu $\mathrm{Hsu}$ in their paper Unity 3D production and environmental perception vehicle simulation platform ${ }^{[3]}$ Have presented as one sketches virtual structures of automobile bodies, are streets and background. Various weather conditions such sunny, rainy and snowy together with artificial variables like sudden crossings by pedestrians, road blocks, vehicle and blind scooter spots and other emergency events which can easily seem, while they go, been considered if one founds the system. The model of the virtual reality which simulates the operation of real cars and unity $3 \mathrm{D}$ is used to integrate the driver into the test screenplay, while the movements of the driver and points of view can serve as a research basis. Different driving screenplays were used to examine how the depth sensors react to raise exactness of the depth sensors if they are applied to real situations during the process of development, and if they are used to check the effects of environmental factors on the scenes.

\subsection{Marking System}

In the paper by NishantSwarnkar, Inderpal Singh Chug, FarazBagwan[1],the score will depend upon the performance of the driver how he performed in the test. For each rule he breaks, a selected number of points will be deducted from the total score. And when the driver passes the minimum barrier required to pass the test, the game will end and result will be displayed that he has failed. Else if the driver doesn't reach the minimum threshold value, he will pass test.

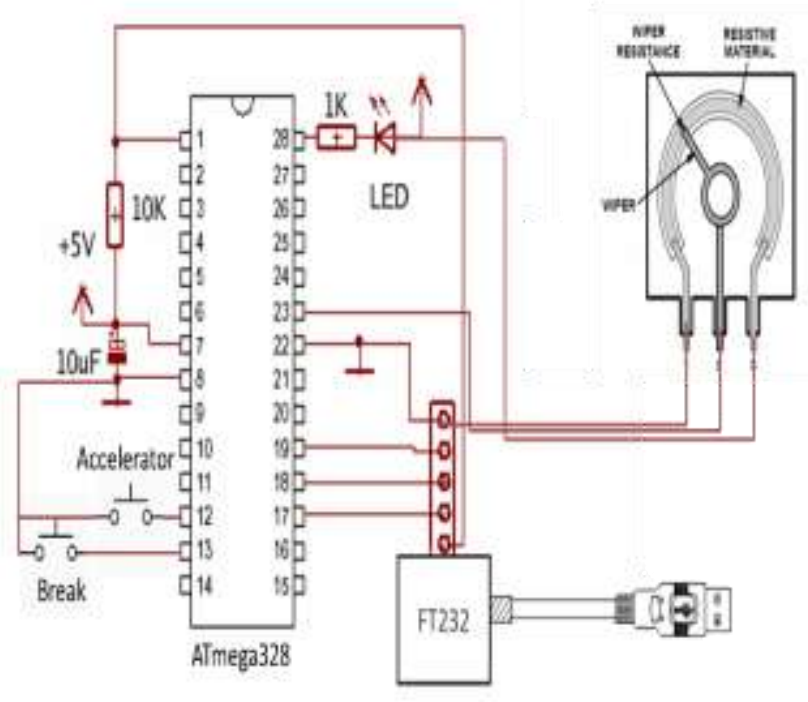

Fig 1: Circuit diagram of automated driver tester.

\section{METHODOLOGY}

\subsection{Hardware Equipments}

1. Arduino UNO

2. Foot Switch 1(Break).

3. Foot Switch 2(Accelerator).

4. Steering Wheel.

5. Rotary Potentiometer.

6. Jumper wire male to female

7. i5 based computer with 4GB hard disk and RAM

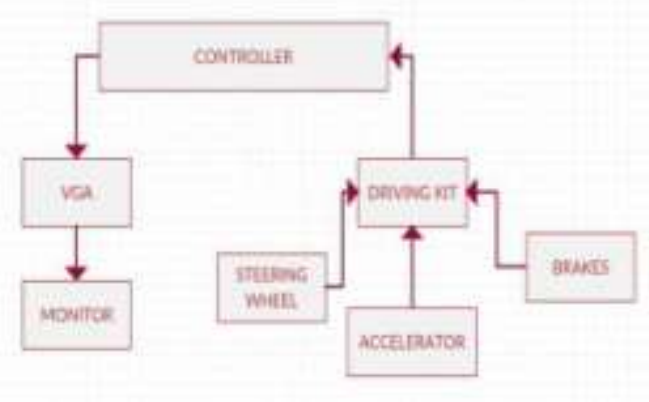

\subsection{Software Equipments}

1. Unity 3D

2. MonoDevelop IDE

3. Arduino IDE 


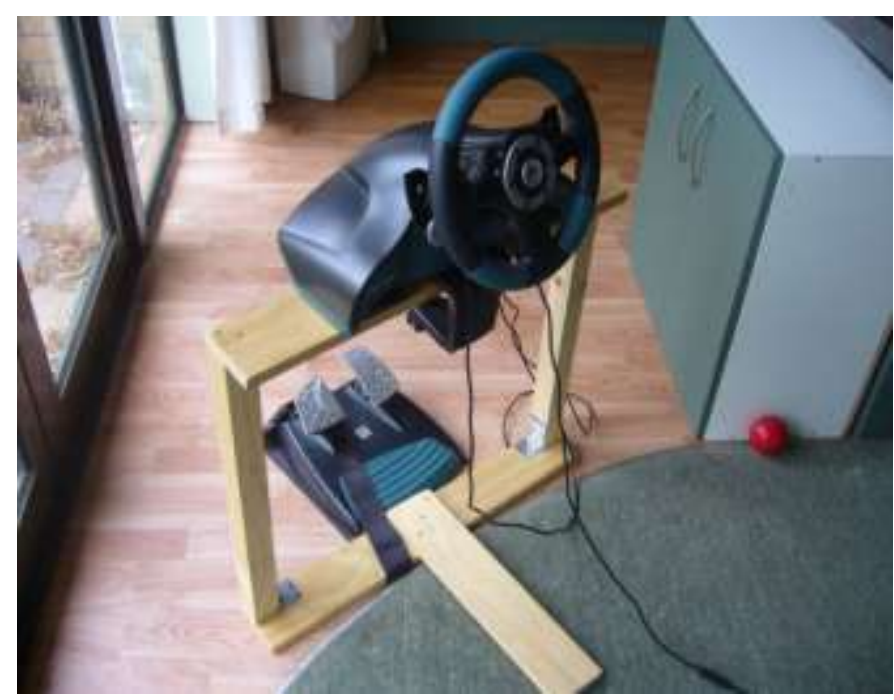

Fig 2: Complete hardware setup

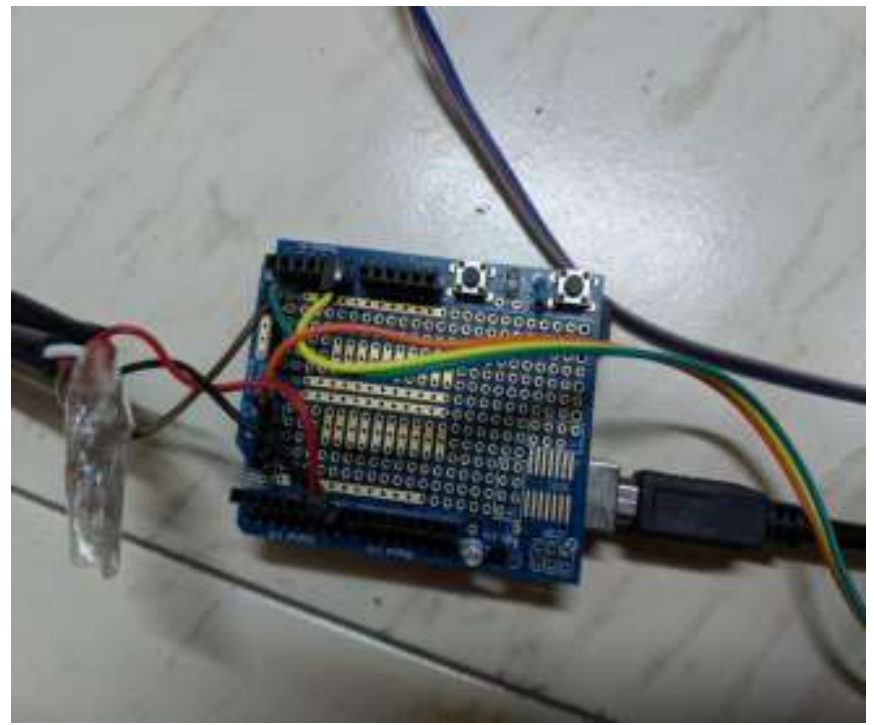

Fig 3: Arduino UNO

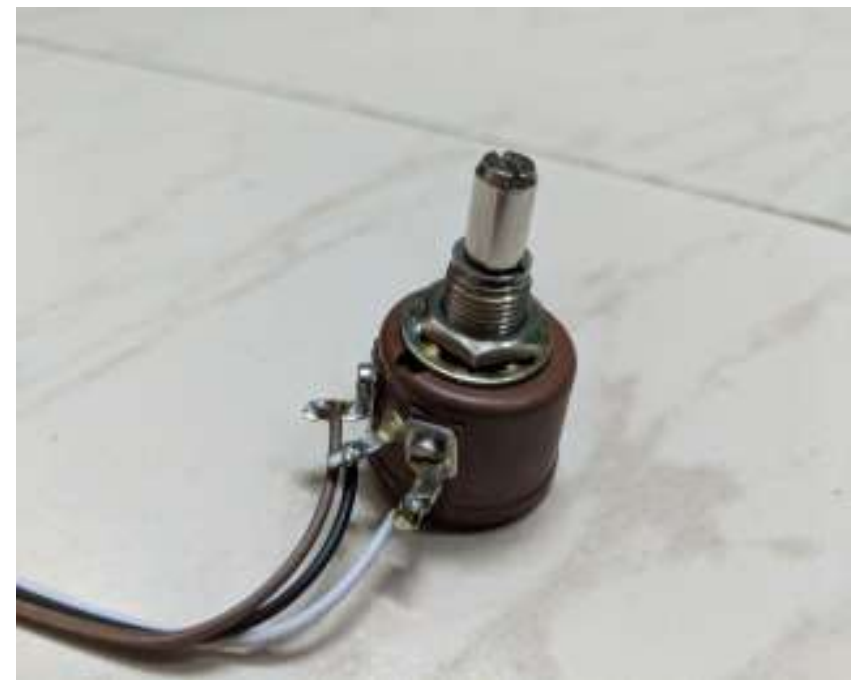

Fig 4: Rotary Potentiometer (Steering Wheel)

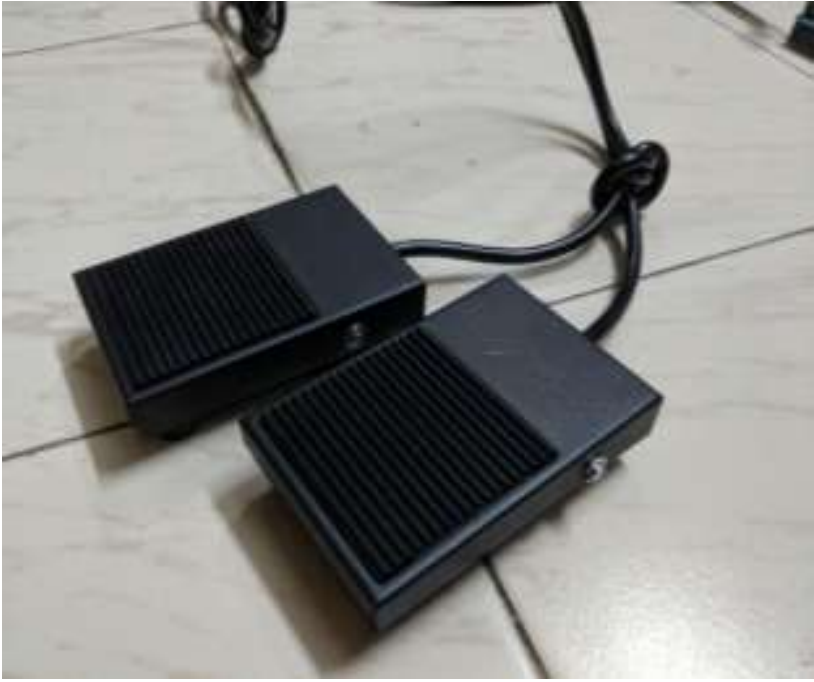

Fig 5: Accelerator and break

\section{FLOWCHART}

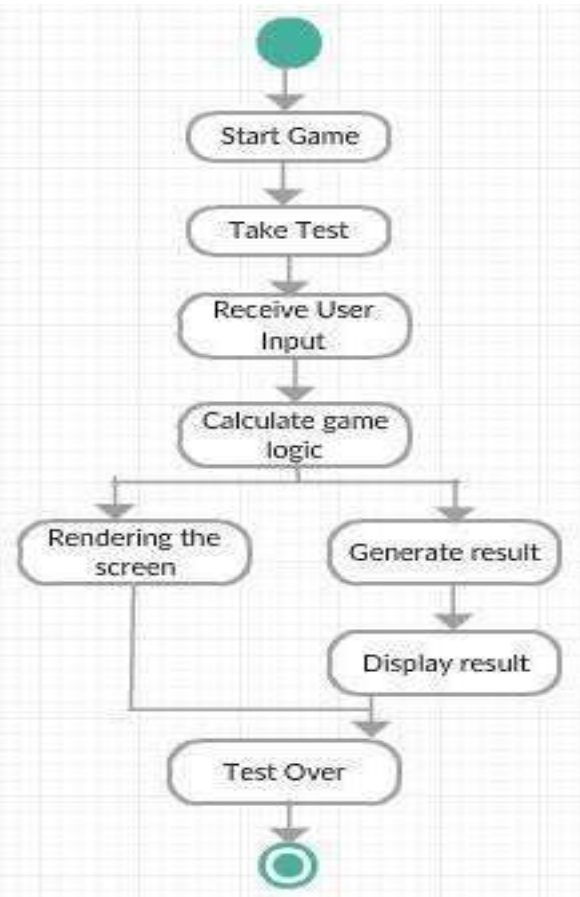

\section{PROPOSED SYSTEM}

\subsection{Virtual Environment}

The virtual environment in the project will be created using a software called as Unity 3D. It will simulate an actual city where there will be all the sceneries and objects that present in a metropolitan city. The design will consists of two lane roads, traffic junctions, highways ,bridges etc. Also effort was dedicated to increasing the degree of accuracy of objects to increase the authenticity of the display. Additionally, vehicle dashboards, traffic lights in intersections, AI physical traffic flows, climate conditions, and collisions will be added to the environment to create a 
more realistic and more informative driving environment simulation platform.

\subsection{Following Steps need to be followed}

Step 1: The user will get a Main menu page where he will have the option to start the test .

Step 2:After starting the test, the user will have to follow the various instructions shown during the test and follow all the rules and regulations while playing. If he commits a mistake, then marks will be deducted depending upon the degree of the mistake hecommits.After all the instructions are followed, the test will be completed.

Step 3: After the test is completed, the result will be displayed that will show his final marks and whether he has passed or failed.

\subsection{Marking System}

Speed Limit: - The speed restriction is $30 \mathrm{~km} / \mathrm{h}$ and if the driver goes beyond a limit of restriction of speed then, he will fail at that time, and 20 points will be subtracted from a total count i.e. 100 points. In this precedent the penalty of restriction of speed included, for example, if the user doesn't control vehicle speed then, then the penalty will be added and if the penalty occurs more than 3 , thus, the user will be failed.

Signboards:- This test case checks, on account of users to follow signs properly or does not follow i.e. during the test there may be signs to turn left or right and do not turn to the left or do not turn right. If user does not follow signs, 20 points will be subtracted.

On Curbs: - In this test case if the users who steers the car out of vision the street then, 30 points are drawn off.

Traffic Signal:- In this test case if then user has broken the traffic signal 30 points are drawn off.

So accordingly software automatically generates the result that user will pass or fail during the test.

\section{CONCLUSION}

Thus in this paper we implemented driving kit using Arduino UNO board and interfaced it with a virtualized driving environment. It will help to improve the quality of drivers on road.

In reality the drivers face some complex situations which are not tested in driving test at RTO. This leads to increased in accidents. The simulator and the game we created comprises these situations thus improving the reflex time of the drivers as they need to respond to the situation as fast as possible

\section{REFERENCES}

[1] NishantSwarnkar, Inderpal Singh Chug, FarazBagwan, GauravThorat, "VR4DT - Virtual Reality for Driving Test", International Journal of Engineering Science and Computing, March 2016

[2] Marvin T. Chan, Christine W. Chan, and Craig Gelowitz "Development of a Car Racing Simulator Game Using Artificial Intelligence Techniques", Hindawi Publishing Corporation International Journal of Computer Games Technology Volume 2015, Article ID 839721.

[3] Proceedings of the IEEE International Conference on Advanced Materials for Science and Engineering IEEE-ICAMSE 2016 Meen, Prior and Lam (Eds), "Unity 3D production and environmental perception vehicle simulation platform"

[4] International Journal of Machine Learning and Computing, Vol. 2, http://www.instructables.com/id/Arduino-UnityRacing-Game/o. 1, February 2012 Game AI: Simulating Car Racing Game by Applying Pathfinding Algorithms Jung-Ying Wang

[5] https://www.hindawi.com/journals/ijcgt/2015/83972 $1 /$

[6] https://docs.unity3d.com/ScriptReference/Help.html.

[7] https://www.arduino.cc/en/Guide/ArduinoUno.

[8] http://www.instructables.com/id/Arduino-UnityRacing-Game.

[9] https://www.youtube.com/watch?v=N9RhSa8oMD8 